\title{
Comparison Between Plasma Properties And Damage Thresholds In Doped Silica Exposed To IR Temtosecond Laser
}

\author{
M. Lancry ${ }^{* 1}$, B. Poumellec ${ }^{* 1}$ and S. Guizard ${ }^{* 2}$ \\ ${ }^{* 1}$ EPCES/ICMMO, UMR CNRS-UPS 8182, Université Paris Sud 11, Bâtiment 410, 91405 Orsay, \\ France \\ E-mail: matthieu.lancry@u-psud.fr \\ ${ }^{* 2}$ Laboratoire des Solides Irradiés/CEA IRAMIS, Ecole Polytechnique, Palaiseau, France
}

\begin{abstract}
Time resolved spectral interferometry shows that both the trapping time of electrons excited in the conduction band is strongly dependent on the doping nature (e.g. Ge, P or F) event for very low concentrations on the order of $1 \mathrm{at} \%$. This is agreement with the birefringence threshold (T2) that exhibits a significant dependence on the used dopants. In contrast, we observed that the permanent isotropic index change threshold (T1) is not significantly dependent on the doping. This indicates that the T1 threshold is not determined by a "critical" excited electron density neither a self-trapped exciton density. Another criterion more appropriate for T1 would be the absorbed energy per pulse and its subsequent transfer to the glass network.
\end{abstract}

Keywords: Silica, Doped silica, Femtosecond laser processing, Ultrafast processes in condensed matter, Laser-induced chemistry, Femtosecond phenomena

DOI:10.2961/jlmn.2012.02.0016

\section{Introduction}

Silica-based glasses are the backbone for many of today's rapidly expanding photonics applications. Current advanced femtosecond laser systems offer a myriad of possibilities to modify glassy media; from surface ablation, annealing, to 3D refractive index modification (positive or negative index change with isotropic or anisotropic properties) depending on the laser parameters [1]. Recently, new unique properties of laser induced glass modifications have been discovered, like chirality [2, 3], directional dependent writing [2-4], oxidation-reduction [5], glass decomposition [6], nanocluster precipitation and shaping [7] and elemental distribution with a sub-wavelength resolution $[6,8]$. To our knowledge, no other technique holds the same potential to realize 3D multi-functional photonic devices, fabricated in a single step in such a wide range of transparent materials. It exhibits enormous potential in the development of a new generation of powerful components for micro-optics, telecommunications, 3D optical data storage, imaging, biophotonic and many more $[5,9,10]$.

From the fundamental point of view, the development of femtosecond laser systems has prompted the investigation of many nonlinear physical phenomena, such as multiphoton induced absorption, plasma formation and avalanche ionisation in glasses. Indeed, in the case of a multiphoton absorption, it is possible to achieve electronic interband transitions (in the conduction band, CB). Specifically, for $800 \mathrm{~nm}$ IR laser and pure silica glass, it needs 6 photons $[11,12]$. In such a case, multiphoton ionization (MPI) leads to free electron. Once the free electron density becomes non-zero, multiphoton absorption (MPA) inducing high energy electrons (typ. 10-30eV [13]), leads to electron-electron collision processes which increase further the free electron density, creating plasma. Although point defects caused by such intense irradiation have been identified in fluorescence, ESR and other studies [14-16], the mechanism of formation of induced modifications in glass is not yet understood.

Although many results have been already achieved in pure silica, only few publications have examined the effect of doping in silica [17-19], however no systematic study has not yet been conducted on the permanent refractive index changes. It is known that it is possible to write in various glasses with a femtosecond laser but we do not know what the chemical dependencies of the plasma properties are and how is it related to the permanent modifications thresholds. Flexibility of multi-component glasses will allow tuning of glass properties including not only photosensitivity but also thermal stability, viscosity, thermal expansion coefficient, etc... It is thus clear that a characterization of the response to the femtosecond irradiation of the key materials already developed and used for photonics applications is required. From an experimental point of view, this issue can be addressed by measuring the permanent damage threshold, the lifetime of photo-excited carriers, the electron plasma density and the self trapped exciton concentration $\left(\mathrm{N}_{\mathrm{STE}}\right)$ as a function of silica doping. Specifically in this paper, we present time-resolved experiments using frequency-based spectral interferometry [11, $20,21]$ together with T1 and T2 thresholds as defined by permanent isotropic index change and form birefringence appearance, in Ge-doped, F-doped and P-F co-doped silica that are typical optical telecommunication glasses.

\section{Experiments}

The experiments were performed in an optical fiber preform plate of $1 \mathrm{~mm}$ thickness and $20 \mathrm{~mm}$ in diameter. The chemical analysis of the preform cross-section was made by EPMA (Electron Probe Micro Analyser) (Fig. 1). The core is mainly germanium-doped silica except the center which is doped predominantly with fluorine. The cladding is equally co-doped with phosphorus and fluorine. The fluctuations seen on the chemical composition profiles are 
well correlated with the MCVD layers. The homogeneous concentration of fluorine in core-cladding is caused by its higher diffusion compared with phosphorus, which as a result marks the layers. This occurs also for the claddingtube interface that contains only fluorine. In the experiments we took advantage of these peculiarities for determining modification thresholds dependence with doping.

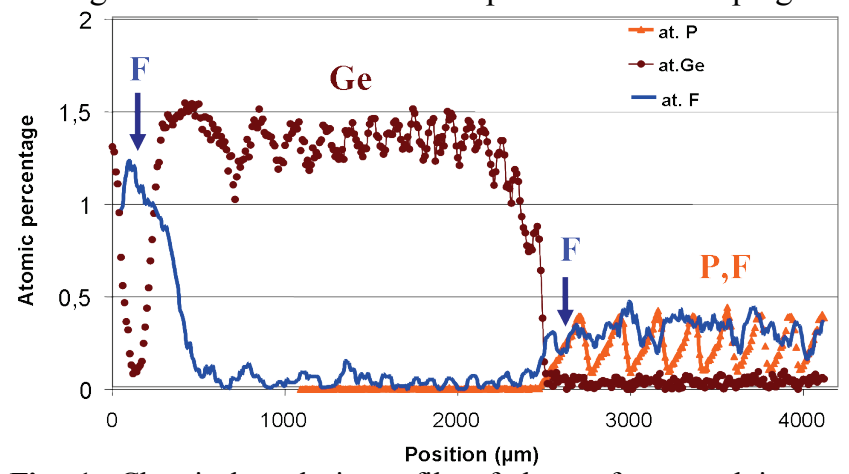

Fig. 1: Chemical analysis profile of the preform used in our experiments. Si and $\mathrm{O}$ contents are not shown. The Ge-doped part is the core of the optical fiber preform. The increase in $\mathrm{F}$ concentration indicates the preform center. Chlorine (in pink) is shown to remain at very low level. The analysis has been made by EPMA.

The laser used in the experiments was a Ti sapphire operating at $800 \mathrm{~nm}, 160 \mathrm{fs}$, and $100 \mathrm{kHz}$ repetition rate. The $\times 20$ microscope objective with NA 0.50 was used for writing in the preform plate. In the experiment 31 lines were drawn $200 \mu \mathrm{m}$ below the entry surface with a pulse energy ranging from $2.6 \mu \mathrm{J}$ down to $55 \mathrm{~nJ}$. The sample was moved along a direction perpendicular (let us say $\mathrm{x}$ ) to the beam tracing continuous lines from the center (Ge doped core) to the peripheral (pure silica substrate) of the fiber preform. The polarization laid along $\mathrm{x}$, i.e. parallel to the sample displacement. The distance between the lines was $50 \mu \mathrm{m}$. After femtosecond laser irradiation, the photo-induced macroscopic structural modifications were inspected by quantitative phase microscopy (QPm) and retardance imaging system (ABRIO, CRI inc.) both based on the Olympus BX51 optical microscope.

For the study of carrier trapping dynamics, a Ti:Sapphire chirped-pulse amplified laser, with a pulse duration $\tau \sim 85 \mathrm{fs}$, wavelength $\lambda_{p}=800 \mathrm{~nm}$. A 0.02 numerical aperture lens was used to focus the pump laser beam and the laser inesnity is estimated around $11 \mathrm{TW} / \mathrm{cm}^{2}$. The spectral frequency domain interferometry (SFDI) uses two identical probe pulses "twin pulses", separated in time by a delay, $\tau$, that is large compared to $\tau_{\text {pulse }}$ and sent to a spectrometer. The perturbation induced by the pump pulse leads to a change of the relative phase between the twin pulses. SDFI uses this shift to measure $\Delta \Phi(t)$, which is a measure in the change in dielectric constant. The detailed experimental setup of a time-resolved interferometric measurement is shown in Ref. [21].

\section{Results}

\subsection{Dynamics of photo-excited carriers}

In Fig. 2 the red curve corresponds to the measured phase shifts at $800 \mathrm{~nm}$ at $300 \mathrm{~K}$ in a undoped $\mathrm{SiO}_{2}$ sample, Gedoped (in blue), F-doped (in black) and P-F co-doped silica (in green) for fixed pump intensity around $11 \mathrm{TW} / \mathrm{cm}^{2}$.
There are several possible origins for the corresponding refractive index change after photo-irradiation of valence electrons in a glass matrix [21]. For the sake of clarity, we will present here an approximate expression Eq. (1) that estimates the phase shift curves, $\Delta \phi(t)$, within an order of magnitude [21] and which is more convenient to identify the contribution of each effect .

$$
\Delta \Phi(t)=\frac{2 \pi}{\lambda} L\left[n_{2} I_{p}(t)+\frac{e^{2}}{2 n_{0} \varepsilon_{0}}\left\{-\frac{N_{C B}(t) \cdot f_{C B}}{m^{*} \omega^{2}}+\sum_{\text {trapkinds }(t r)} \frac{N_{t r}(t) \cdot f_{t r}}{m\left(\omega_{t r}^{2}-\omega^{2}\right)}\right\}\right](1)
$$

The first part of the curves contributes positively (analogous to a convex lens) to the phase shift because the nonlinear index is positive at the probe wavelength. It is observed in all materials and will occur as long as the pump and the probe pulses overlap in time within the sample. We observed that it is not significantly modified in doped silica when compared to $\mathrm{SiO}_{2}$. This is in agreement with the change in $\mathrm{n}_{2}$ that is known to be less than $10 \%$ [22] at our doping level and is quite negligible. The delay corresponding to the maximum value of this term has been used to define the zero of our time delay curves. Notice that the FWHM is much larger than expected from the convolution of the probe and the pump pulse duration $(\approx 60 \mathrm{fs})$. This temporal broadening (estimated to $85 \mathrm{fs}$ ) is likely due to the group-velocity dispersion in the optics during the beam pathway.

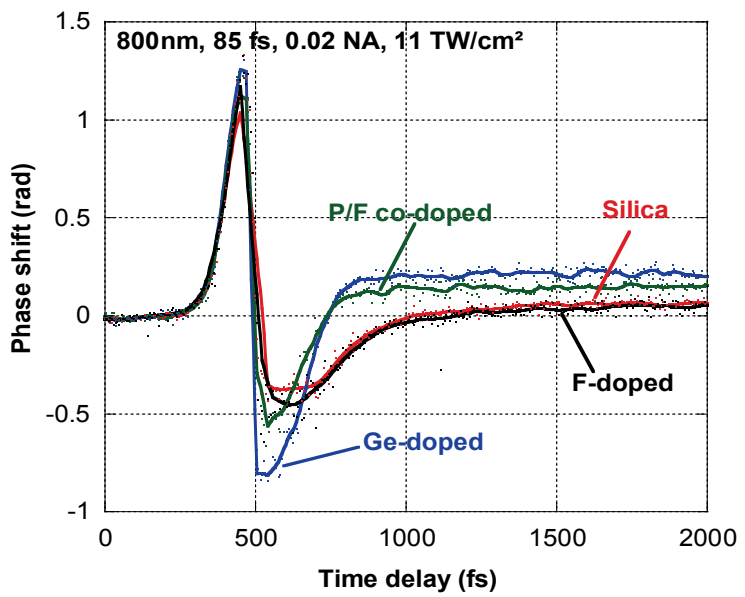

Fig. 2 Phase shift as function of time delay measured in $\mathrm{SiO}_{2}$ (in red), F-doped (in black), P/F co-doped (in green) and Ge-doped $\mathrm{SiO}_{2}$ (in blue) for the same pump intensity of $11 \mathrm{TW} / \mathrm{cm}^{2}$. The probe wavelength is $800 \mathrm{~nm}$ and the sample temperature is $300 \mathrm{~K}$. The full lines correspond to guides for eye.

The second part is proportional to $N_{\mathrm{CB}}$, the density of electrons in the conduction band (CB) and is always negative. This term accounts for the observed negative phase shift shown in the curves. Notice it is significantly larger than in doped silica and especially in Ge-doped silica.

The subsequent evolution of the phase shift evolves toward a positive value. This last part stands for the density of trapped electrons $N_{t r}$. Its sign is determined by the relative energy values of $\omega_{\mathrm{tr}}$ (the excitation energy of the trapping site) and $\omega$. For the experiment described here, the observation of a positive phase shift at the end of the curves indicates that there is trapping of electrons. The transient absorption band $\omega_{t r}$ associated with the trapping site correspond to higher frequency than the probe beam frequency $\left(\omega_{t r}>\omega\right) . f_{\text {tr }}$ is the oscillator strength for the trap level. Consequently the phase shift, $\Delta \Phi_{\infty}$, measured at a 
sufficiently large delay ( $\approx 2 \mathrm{ps}$ ) after the laser pulse allows to calculate the density of electrons that has been previously excited, $N_{t r}$, in the solid at the end of the laser pulse [11, 21]. We observe that $\Delta \Phi_{\infty}$ is at least 4 times higher in Gedoped silica and 3 times higher in $\mathrm{P} / \mathrm{F}$ co-doped silica when compared to pure silica and F-doped silica. Surprisingly we also observe that the time to get a positive phase shift is much smaller in Ge-doped and $\mathrm{P} / \mathrm{F}$ co-doped $\mathrm{SiO}_{2}$. This indicates that electrons trapping time $\tau_{\text {tr }}$ is significantly smaller in these materials.

\subsection{Permanent modifications thresholds}

As described in the introduction, we define the first damage threshold $\mathrm{T} 1$ as the pulse energy that appears a permanent isotropic index change. Notice that we performed multiple (typ. 1000) pulses threshold measurements. Usually, it gives rise to a converging lens effect and thus to a higher luminosity. The second threshold $\mathrm{T} 2$ is (as defined in this paper) the pulse energy above which a strong linear birefringence appears and can be easily seen in crossed Nicol polarizer configuration or using retardance imaging system as shown in Figs 3 and 4.

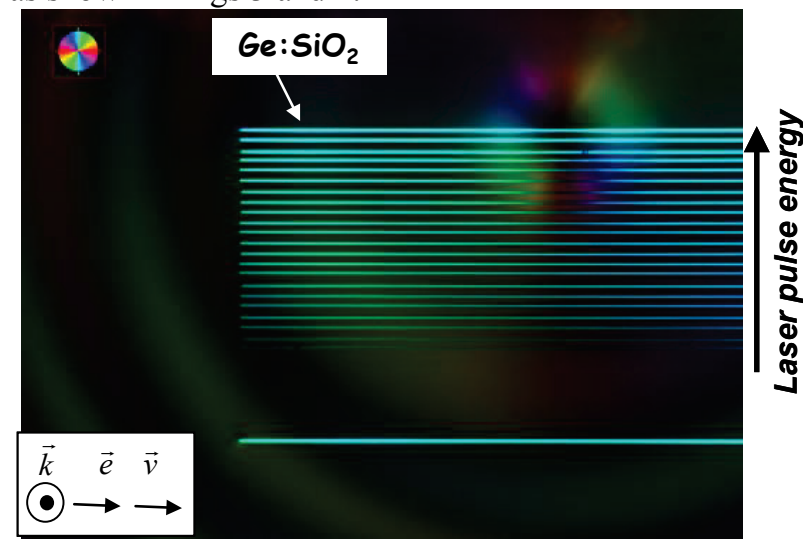

Fig. 3: Quantitative birefringence cartography in optical fiber Ge-doped preform core. The blue lines correspond to various writing pulse energies ranging from $55 \mathrm{~nJ}$ up to $2.6 \mu \mathrm{J}$. The color scale indicates the slow axis orientation.

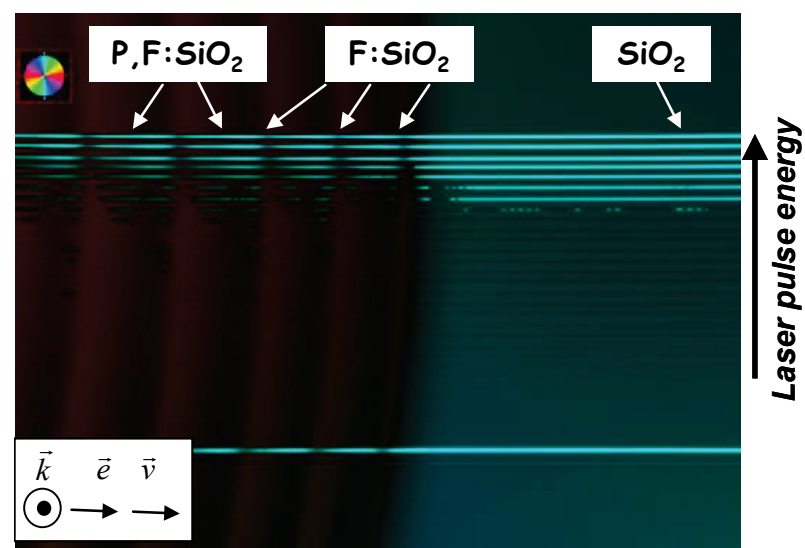

Fig. 4: Quantitative birefringence cartography in optical fiber F$\mathrm{P}$ co-doped cladding and pure silica tube. The blue lines correspond to various writing pulse energies ranging from $55 \mathrm{~nJ}$ up to

$2.6 \mu \mathrm{J}$. The color scale indicates the slow axis orientation.

Firstly, the femtosecond laser written line structures were inspected using the retardance imaging system. The color scale (i.e. disk in the up-left corner) indicates the orientation of the birefringence slow axis (the large index neu- tral axis) while the magnitude (i.e. color intensity) is correlated to the retardance level. The appearance of bright blue lines indicates the anisotropic modification (above T2) (Fig. 3). Quantitative characterization (Figs. 3 and 4) with the Abrio system revealed some color contrast in the background, which is due to residual radially symmetric stress field related to inhomogeneous thermal expansion coefficient distribution induced by the MCVD manufacturing process $[23,24]$. This effect is especially noticeable around the preform center, where the birefringence is particularly large due to the strong chemical contrast. Then, concerning the lines, it can be seen that the slow axis is perpendicular to the lines' orientation (i.e. blue color). Notice that these lines have been written with laser polarization parallel to the scanning direction. If we write lines with a laser polarization perpendicular to the scanning direction, the lines will appear in red color. This confirms that the principal axes are determined by the laser polarization.

By correlating, the energy damage thresholds determined from Figs 3 and 4 as a function of material chemical composition profile (as shown in Fig. 1), we are then able to determine the $\mathrm{T} 2$ threshold according to the doping. Comparing with silica $(0.31 \mu \mathrm{J} /$ pulse here $)$, for 1.5 at $\%$ Gedoped silica the threshold T2 is several times smaller $(0.14$ $\pm 0.05 \mu \mathrm{J} /$ pulse) as shown in Fig. 3. For a silica doped with 0.3 at $\%$ of fluorine (see arrows in Fig. 4), the second threshold T2 is close to $1.20 \pm 0.05 \mu \mathrm{J} /$ pulse. The addition of 0.3 at $\%$ of phosphorus to 0.3 at $\%$ F-doped silica (see left arrows in Fig. 4) degrades the second damage threshold from 1.20 to $0.25 \pm 0.05 \mu \mathrm{J} /$ pulse. Under optical microscope working in transmission mode the lines written below the second threshold could be also detected. The contrast in the optical image arises from an isotropic variation of refractive index. In this way, we deduce that the first threshold is not influenced significantly by the doping and is always fixed at $0.095 \pm 0.005 \mu \mathrm{J} /$ pulse.

\section{Discussion}

\subsection{Dynamics of photo-excited carriers}

In the following kinetics scheme, the density of excitations and the Coulomb force between electron and hole, along with the absence of sufficient existing traps and electrons donors in silica, leads us to consider that they are interdependent and hence can be considered as a single species. More details can be found in Ref. [25]. Then for the sake of clarity, we will present here an interpretation based on the following mechanism simplification:

$$
\begin{aligned}
& \text { during the pulse } \begin{array}{l}
X(\text { Si }) \stackrel{\mathrm{MPI}}{\longrightarrow} X(\mathrm{Si})^{+}+e_{B C} \\
X(\text { dopant }) \stackrel{\mathrm{MPI}}{\longrightarrow} X(\text { dopant })^{+}+e_{B C}
\end{array} \\
& \text { after the pulse } \mid \begin{array}{l}
e_{C B}+X(\text { Si })^{+} \stackrel{\text { electronic trapping in a few 100fs }}{\longrightarrow} S T E(\text { Si }) \\
e_{C B}+X(\text { dopant })^{+} \stackrel{\text { electronic trapping in a few 100fs }}{\longrightarrow} S T E \text { (dopant) }
\end{array}
\end{aligned}
$$

where $X(\mathrm{Si})$ and $X$ (dopant) means sites around oxygen near $\mathrm{Si}$ and the involved dopant (e.g. Ge or P), respectively, $X$ is a regular site of glass near the oxygen atoms and $X^{+}$is the corresponding Self Trapped hole (STh). Self Trapped Excitons $(S T E)$ are formed in less than one ps. Indeed, it is now well known that ionizing radiation produces self-trappedexciton (STE) in $\mathrm{SiO}_{2}$ and slightly doped silica [26-30]. In $\mathrm{SiO}_{2}$ and $\mathrm{Ge}$-doped $\mathrm{SiO}_{2}$, besides radiative recombination, they may relax into E' and NBOHC [20], labelled as defects above. Then E' ( $\mathrm{Si}$ or $\mathrm{Ge}$ ) and $\mathrm{NBOHC}$ can recom- 
bine into $\mathrm{T}-\mathrm{ODC}$ (II) $(\mathrm{T}=\mathrm{Si}$ or $\mathrm{Ge})$ and POR (Peroxy linkage $\mathrm{Si}-\mathrm{O}-\mathrm{O}-\mathrm{Si}$ or peroxy radical $\mathrm{Si}-\mathrm{O}-\mathrm{O} \bullet$ ). There is also another relaxation channel that can produce T-ODC(I) and interstitial oxygen. However, the production of permanent defects is less than $1 \%$ of the regular relaxation [15]. On the other hand, since both would be formed a few ns after the excitation and probe pulses, they are not seen in the present experiment since measurements are taken during the first picoseconds and thus sensitive to transient processes only. The experiment therefore neglects the actual possibility for STE to generate defects such as SiODC that can potentially act as further electron sources, and $\mathrm{Si}-\mathrm{ODC}{ }^{+}$ centres as trapping sites. We have checked their contribution. By performing the measurement after $2 \times 10^{4}$ pulses these contributions were not detected. Further, we did not detect any difference also between Infrasil or Suprasil silica [31] which indicates that if these defects are generated they play a negligible role in phase shift at this concentration level.

In the following $f_{\mathrm{CB}}$ is taken equal to $1 . m^{*}$ is the electron effective mass in the CB. For low energy electrons $m^{*}$ is close to 0.5.m assuming a parabolic band [32]. However it has been shown that for higher electrons energy, this value increases and follows a trend to saturation around $\mathrm{m}$. In addition an averaging is made on the electrons according to their kinetics energy [33]. In the following we will thus assume that $\mathrm{m}^{*}=\mathrm{m}$ and we will consider similar values whatever the doping may be. From the literature an energy of $4.6 \mathrm{eV}$ and $5.7 \mathrm{eV}$ are commonly associated to $\omega_{\mathrm{trSi}}$ for amorphous pure silica [27, 30]. In the case of Ge-doped silica, we found a transient absorption band related to a STE at an energy of $4.1 \mathrm{eV}$ associated to $\omega_{\text {trGe }}$ [30]. Practically, we will consider in each case only the dominant term, i.e., the one which is the closest to resonant transitions for our probe wavelengths. In this model, the optical absorption spectra associated with electrons trapped in the band gap are represented by single absorption lines. In other words, $f_{t r S i}$ and $f_{t r G e}$ must also be considered as an adjustable effective parameter because, at least in principle, several transitions with different probabilities are possible. The multiphoton absorption coefficients $\sigma_{6}$ and $\sigma_{5}$ will be also considered as adjustable parameters.

First, we simulated the $\mathrm{SiO}_{2}$ curve using the approach described in Ref. [25] with three adjustable parameters $\left(f_{t r S i}, \tau_{t r S i}\right.$ and $\left.\sigma_{6}\right)$ and without the terms related to the presence of doping. We found electron trapping time $\tau_{\text {tr }}$ of 155 \pm 5 fs and an oscillator strength around $0.3 \pm 0.1$ in agreement with previous publication [21]. From the simulation, we obtain an average density $N_{t r S i}$ around $9.10^{18} \mathrm{~cm}^{-3}$ close to the OB threshold in pure silica. As the excitation density is not large enough to consider two excitations at the same location, electron-hole pairs can be considered again as preserved entities. So in doped samples, we will speculate that the kinetics around $\mathrm{Si}$ or around the dopant $(\mathrm{Ge}$, or P) is independent. Furthermore, if we assume in first approximation that silica constants $\left(\sigma_{6}, \tau_{\mathrm{trSi}}\right.$ and $\left.\mathrm{f}_{\mathrm{trSi}}\right)$ are not changed significantly by the doping, we can deduce the parameters attached to the involved dopant and then compare the concentrations of the different species. We have thus simulated the curves related to doped with three ad- justable parameters $\left(f_{\text {trdopant }}, \tau_{\text {trdopant }}\right.$ and $\left.\sigma x\right)$. In Table 1 , we present a summary of the set of parameters that fits satisfactorily the measured phase shifts in Fig. 2. Notice that the obtained results for the multiphoton cross-section are in good agreements with the results already reported in the literature [33].

\begin{tabular}{|c|c|c|c|c|c|}
\hline & & $\mathrm{SiO}_{2}$ & Ge-doped $\mathrm{SiO}_{2}$ & $\begin{array}{c}\mathrm{P} / \mathrm{F}- \\
\text { doped } \\
\mathrm{SiO}_{2} \\
\end{array}$ & F-doped $\mathrm{SiO}_{2}$ \\
\hline \multicolumn{6}{|l|}{$\begin{array}{c}\begin{array}{c}\text { Fixed parame- } \\
\text { ters }\end{array} \\
\end{array}$} \\
\hline $\begin{array}{c}\text { Nonlinear } \\
\text { refractive index } \\
\left(\mathrm{cm}^{2} / \mathrm{W}\right)\end{array}$ & $n_{2}$ & $2.76 .10^{-16}$ & $\begin{array}{c}(2.76+0.097 . x) \cdot 10^{-} \\
16, \text { where } \mathrm{x} \text { is } \mathrm{Ge} \\
\text { in } \mathrm{mol} \%\end{array}$ & NA & $\begin{array}{c}(2.76+1.03 . x) .10 \\
16, \text { where } x \text { is } F \\
\text { in } w \%\end{array}$ \\
\hline $\begin{array}{l}\text { Initial valence } \\
\text { electron density } \\
\left(\mathrm{cm}^{-3}\right)\end{array}$ & $N_{0}$ & $2.2 .10^{22}$ & $2.2 .10^{22}$ & $2.2 .10^{22}$ & $2.2 .10^{22}$ \\
\hline $\begin{array}{l}\text { Order of the } \\
\text { multiphoton } \\
\text { process }\end{array}$ & $n$ & 6 & 5 & 5 & 6 \\
\hline $\begin{array}{c}\text { Oscillator } \\
\text { strength for the } \\
\text { CB }\end{array}$ & $f_{C B}$ & 1 & 1 & 1 & 1 \\
\hline $\begin{array}{l}\text { Electron effec- } \\
\text { tive mass in the } \\
\text { CB }\end{array}$ & $m^{*}$ & $\mathrm{~m}$ & $\mathrm{~m}$ & $\mathrm{~m}$ & $\mathrm{~m}$ \\
\hline $\begin{array}{c}\text { Trap level energy } \\
(\mathrm{eV})\end{array}$ & $\omega_{t r}$ & 4.6 & 4.1 & 4.6 & 4.6 \\
\hline \multicolumn{6}{|l|}{$\begin{array}{c}\text { Adjustable } \\
\text { parameters }\end{array}$} \\
\hline $\begin{array}{l}\text { Electron trapping } \\
\text { time }(\mathrm{fs})\end{array}$ & $\tau_{t r}$ & $155 \pm 5$ & $75 \pm 5$ & $85 \pm 5$ & $165 \pm 5$ \\
\hline $\begin{array}{l}\text { Oscillator } \\
\text { strength for the } \\
\text { trap level }\end{array}$ & $f_{t r}$ & $0.3 \pm 0.1$ & $0.3 \pm 0.1$ & $0.4 \pm 0.1$ & $0.4 \pm 0.1$ \\
\hline \multirow{5}{*}{$\begin{array}{l}\text { Multiphoton } \\
\text { cross section }\end{array}$} & $\sigma$ & $\sigma_{6}=$ & $\sigma_{5}=(1.8 \pm$ & $\sigma_{5}=$ & $\sigma_{6}=(1 \pm$ \\
\hline & & $(4.5 \pm$ & $0.2) \cdot 10^{-55} \mathrm{~s}^{-}$ & $(2.0 \pm$ & $0.2) \cdot 10^{-68} \mathrm{~s}^{-}$ \\
\hline & & $0.2) .10^{-69}$ & ${ }^{1} \mathrm{~cm}^{10} \mathrm{~W}^{-5}$ & $0.2) .10^{-}$ & ${ }^{1} \mathrm{~cm}^{12} \mathrm{~W}^{-6}$ \\
\hline & & $\mathrm{s}^{-}$ & & ${ }^{55} \mathrm{~s}^{-}$ & \\
\hline & & ${ }^{1} \mathrm{~cm}^{12} \mathrm{~W}^{-6}$ & & ${ }^{1} \mathrm{~cm}^{10} \mathrm{~W}^{-5}$ & \\
\hline
\end{tabular}

Table 1: Set of parameters used in the simulation for $\mathrm{SiO}_{2}$ and doped $\mathrm{SiO}_{2}$.

From the simulation procedure described above, we can extract the average electron trapping time $\tau_{\text {tr }}$ and the density $\boldsymbol{N}_{t r}$. We found $155 \pm 5 \mathrm{fs}$ in pure silica, $165 \pm 5$ in Fdoped silica, $85 \pm 5 \mathrm{fs}$ in $\mathrm{P} / \mathrm{F}$ co-doped silica and $75 \pm 5 \mathrm{fs}$ in Ge-doped silica. Surprisingly, doping $\mathrm{SiO}_{2}$ with only 1.5 at $\%$ of $\mathrm{Ge}$ or 0.3 at $\%$ of $\mathrm{P} / \mathrm{F}$ leads to a significantly faster electron trapping time when compared to pure $\mathrm{SiO}_{2}$, as seen directly in Fig. 2.

To explain our observations in Ge-doped silica, we have thus to consider that there is an additional trapping center involving Ge atom (e.g. a Ge STE that will further relax into $\mathrm{Ge}(1)$ that is a Germanium Electron Center absorbing around 280nm [34, 35]). This additional pathway leads to a significantly faster trapping when compared to pure silica or F-doped silica. Indeed, the trapping rate on a $X(G e)$ containing hole is shown to be faster ( $\tau_{\text {trGe }} \approx 75 \mathrm{fs}$ ) than in silica $\left(\tau_{\text {trSi }} \approx 155 \mathrm{fs}\right)$. In addition, the reaction constant of the second equation is much larger than the first one as the excitation of an electron from valence band to Ge $4 \mathrm{~s}-\mathrm{p}$ orbitals needs only 5 photons even if the $X(\mathrm{Ge})$ density is 10 times smaller than $X(\mathrm{Si})$ density. This leads to a slightly higher excitation density when compared to pure silica. Indeed, we found that the density $N_{t r G e}$ is around 2.2.10 ${ }^{19}$ $\mathrm{cm}^{-3}$ in Ge-doped silica and $N_{\text {trP }}$ is around $1.5 .10^{19} \mathrm{~cm}^{-3}$ in $P$-doped silica.

A similar explanation can be put forward in the case of $\mathrm{P} / \mathrm{F}$ co-doped silica. Indeed, the localized states due to the disordered structure manifest themselves as an optical absorption tail also in P-doped silica. For example, it has been reported that phosphorus doping does not result in any pro- 
nounced absorption features at $5 \mathrm{eV}$ but produces a weak band centred at $4.6 \mathrm{eV} \mathrm{[36]} \mathrm{and} \mathrm{a} \mathrm{strong} \mathrm{one} \mathrm{around} \mathrm{7.6eV.}$ Substitutional $\mathrm{P}$ in quartz and silica has been also studied by EPR [36, 37]. Three different signals have been observed in EPR spectra. P1 (three-coordinated P atom with one unpaired electron, $\left[\mathrm{O}_{3} \equiv \mathrm{P}^{\bullet}\right]^{+}$, energy cost $7.6 \mathrm{eV}$ [38]) and $\mathrm{P} 4$ (two-coordinated $\mathrm{P}$ atom with one unpaired electron, $\left[\mathrm{O}_{2}=\mathrm{P}^{\bullet}\right]^{0}$, energy cost $\left.0.7 \mathrm{eV}[38]\right)$ are trapping sites. The presence of such trapping sites can thus easily explain the faster trapping kinetic that we extracted. In contrast P2 (four-coordinated $\mathrm{P}$ atom with one unpaired electron, $\left[\mathrm{O}_{2}=\mathrm{P}^{\circ}=\mathrm{O}_{2}\right]^{0}$, energy gain $\left.3.5 \mathrm{eV}[38]\right)$ is an additional source of electrons leading to a slightly higher free electrons density $\mathrm{N}_{\mathrm{CB}}$ when compared to silica. In addition as for $\mathrm{GeO}_{2}$ doping, the presence of this additional near-edge absorption will tend to extend the apparent absorption edge tail leading likely to a 5 photons absorption process (as for Ge-doped silica [25]) instead of 6 photons for pure silica.

In contrast to $\mathrm{Ge}$ and $\mathrm{P}$ that are network formers, fluorine is a network modifier. A small quantity (up to $1 \mathrm{~mol} \%$ ) of fluorine "blueshifts" the apparent absorption edge of pure silica [39]. In [39], Skuja et al. reported that at least 1/3 of the disorder-induced broadening is reduced in F-doped silica. They confirm that the main mechanism of VUV transparency increase in F-doped silica is the removal of strained $\mathrm{Si}-\mathrm{O}-\mathrm{Si}$ bonds by a reduction of 4- and 3membered rings [40] and mainly via formation of nonabsorbing Si-F bonds [32, 39, 41]. In addition, recent studies have also shown that the formation of $\mathrm{Si}-\mathrm{F}$ groups allows the reduction of defect precursors [42-44], such as strained bonds from which are likely to be generated defects such as E' and non-bridging oxygen hole center (NBOHC) that absorb in visible and UV spectral range. Doping silica with Fluorine results thus in non-significant changes in the plasma kinetics when compared to pure silica.

\subsection{Modification thresholds}

To summarize, we have measured the chemical dependence of isotropic and anisotropic index change and in particular the thresholds T1 and T2 in MCVD perform taking advantages of its Ge, $\mathrm{P}, \mathrm{F}$ profile. In such sample, we have written a series of lines with pulse energies ranging from $55 \mathrm{~nJ}$ to $2.6 \mu \mathrm{J}$ from the center (Ge doped core) to the peripheral (substrate pure silica tube). Then, we have observed the index change by means of transmitted natural, polarized light, birefringence quantitative measurements and QPm. Correlating the chemical profile (Fig. 1) with our measurements; we have thus deduced the variation of the T1 and T2 energy thresholds. Table 2 summarizes these results. T1 is independent on doping, T2 decreases strongly on $\mathrm{Ge}$ or $\mathrm{P}$ doping but it increases on $\mathrm{F}$ doping.

An important role in the interaction process might be played by self-focusing (SF) [45], in particular when the pulse energy overcomes the SF threshold and a filament appears. Self-focusing is likely to occur at the intensity we use in the interferometry measurements. However this effect needs some propagation distance to become observable. Since we are probing a thin layer below the surface (of the order of $70 \mu \mathrm{m}$ ), we are convinced that the beam profile has not been significantly modified at this depth. For the same reason it is difficult to compare self-focusing in different samples in our set-up, since we do not observe a strong influence of self-focusing or other propagating effect. We plan to study more carefully the effect of propagation in the future, by probing at different depth within the sample. Anyway, for comparison purpose, the theoretical SF values are reported in the right column of Table 2. As it is well known self-focusing is defined by the linear and the nonlinear $n_{2}$ refractive index. It should also be noted that selffocusing depends only on the peak power in the beam [45], but not intensity; thus the critical self-focusing threshold does not depend on the focusing strength. As it can be seen, $n_{2}$ increases only slightly with the presence of either Ge or F [22] when compared to pure $\mathrm{SiO}_{2}$. The self-focusing effect is roughly the same whatever the doping (at the level used in our sample). On the contrary, the appearance of a linear birefringence is strongly dependent on doping. Especially in F-doped silica, the threshold energy for selffocusing is 3 times lower than T2, indicating that selffocusing does not play a detectable role in the formation of index anisotropy.

\begin{tabular}{cccc}
\hline Composition & $\begin{array}{c}\text { T1 thresh- } \\
\text { old }\end{array}$ & $\begin{array}{c}\text { T2 thresh- } \\
\text { old }\end{array}$ & $\begin{array}{c}\text { SF thresh- } \\
\text { old }\end{array}$ \\
\hline $\mathrm{SiO}_{2}$ & 0.095 & 0.31 & 0.35 \\
& $\mu \mathrm{J} /$ pulse & $\mu \mathrm{J} /$ pulse & \\
\hline 1.5 at\% $\%{\mathrm{Ge}: \mathrm{SiO}_{2}}^{0.095}$ & 0.15 & 0.39 \\
& $\mu \mathrm{J} /$ pulse & $\mu \mathrm{J} /$ pulse & \\
\hline 0.3 at $\% \mathrm{~F}: \mathrm{SiO}_{2}$ & 0.095 & 1.20 & 0.39 \\
& $\mu \mathrm{J} /$ pulse & $\mu \mathrm{J} /$ pulse & \\
\hline 0.3 at $\% \mathrm{~F}$ and 0.3 at $\%$ & 0.095 & 0.25 & Unknown \\
$\mathrm{P}: \mathrm{SiO}_{2}$ & $\mu \mathrm{J} /$ pulse & $\mu \mathrm{J} /$ pulse & \\
\hline
\end{tabular}

Table 2: Dependence on $\mathrm{T} 1$ and $\mathrm{T} 2$ thresholds on doping. For sake of comparison we also add the self-focusing threshold SF that is directly related to the non-linear index $\mathrm{n}_{2}$.

Despite the mechanism of photo-induced modification formation in silica glass is not understood, T1 (i.e. appearance of permanent isotropic index change) was suggested to be related on point defects distributed isotropically or with a weak anisotropy [46]. Indeed, point defects such as NBOHC (Non Bridging Oxygen Hole Center), SiE' (Si dangling bonds), peroxy linkage or radical, and interstitial oxygen (atoms or molecules), bond angle tetrahedral rotation [47], density change [48] have been identified in luminescence, electron paramagnetic resonance (EPR), Raman spectroscopy and other studies $[14,16]$ of fused silica. In contrast, T2 is defined by the appearance of a new nanostructure (i.e. nanoplanes of low atomic density) and should be related with some kind of phase transition. Indeed, we have proved recently that nanoplanes consist of porous matter produced by a decomposition of $\mathrm{SiO}_{2}$ into $\mathrm{SiO}_{2(1-\mathrm{x})}+\mathrm{x} \cdot \mathrm{O}_{2}[6]$

\section{T1 interpretation}

For understanding the independence of $\mathrm{T} 1$ on doping for large number of pulses (typ. 1000 pulses) we have to analyze precisely what is the basis of $\mathrm{T} 1$, which is defined here through the appearance of permanent isotropic index change. In other papers [45] it is defined through attenuation permanent variation in the near IR but it is actually the same threshold. What we call threshold corresponds to the clear intersection between two lines in a given plot of the property according to the pulse energy. Permanent isotropic index changes or attenuation decrease are both arising from extended glass modifications. On the other hand, in the first femtoseconds after the pump pulse beginning, the energy 
absorbed from the femtosecond laser pulse leads to formation of excited electrons that relax first into self-trapped excitons (STE) on the 100 femtosecond timescale and then annihilate or transform into point defects later on [31], following the reaction scheme sketched for the Ge-doped silica case:

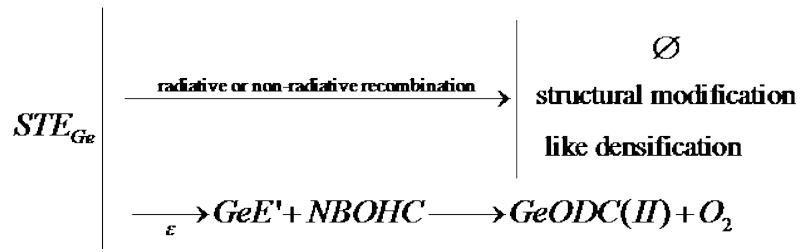

Glass modifications are thus the result of STE relaxation. Three ways of STE decay are possible: a simple radiative recombination with a photon emission (lifetime of nanoseconds at room temperature [31]) or a non-radiative one by coupling with lattice phonons. STE can also transform into point defects (e.g. GeODC(II)) [20] but its yield is small i.e. less than $1 \%$ of the STE concentration [49]. On the other hand, the permanent attenuation in the IR lies in a wavelength range where there is no point defect absorption [50] and thus the transparency loss is thus resulting of long or medium range structural modifications but not from point defects. We speculate that such glass transformation results from non-radiative relaxation of excited electrons by coupling with the lattice. This coupling corresponds to an increase of non-equilibrium temperature of the lattice that can reach a few thousands degrees in the illuminated area just limited by thermal conduction. The temperature increase during a time long enough can lead to glass melting and to a change of average disorder i.e. a change of glass fictive temperature [51]. We can define this temperature $\left(T_{c}\right)$ by saying that the relaxation time defined by the ratio between the viscosity $(\eta)$ and the shear modulus $(G)$ [52] should be smaller than the time during which the matter overcome this temperature. This can be written as:

$$
\eta\left(T_{c}\right) / G\left(T_{c}\right)=\delta t\left(T>T_{c}\right)
$$

On the other hand, the time-temperature profile of the irradiated area is mainly defined by the amount of energy density released in the lattice during the laser pulse divided by the specific heat capacity and the glass density [53]. This energy comes from excited electron-relaxation and not directly from pump pulse that is not completely absorbed by the matter through electron excitation. As a matter of fact, the energy releases by a STE is at least equal to $E_{g}$ but less than $2 \mathrm{E}_{\mathrm{g}}$ as no avalanche is detected in our conditions $[20,25]$. Let assume that this energy is roughly the same whatever the doping (so $\mathrm{a}=$ constant and $1<\mathrm{a}<2$ ). So, finally the released energy density (W) is expressed as the product of (a), of the forbidden energy gap $E_{g}$ of the glass and of the STE concentration at the end of the pulse i.e. $W=a \cdot E_{g} \cdot[S T E]$.

The STE concentration is obtained by time integration of the MPI (multiphoton ionization) rate. It yields approximately $[S T E]=\sigma_{n} I_{t h}^{n} \tau N_{O}$ where $\sigma_{\mathrm{n}}$ is the multiphotons absorption cross section, $\tau$ is the duration of the pulse, $\mathrm{N}_{\mathrm{O}}$ the state density at the top of the valence band and $I_{\text {th }}$ the light intensity threshold corresponding to T1 threshold energy. It comes thus:

$$
W=a \cdot E_{g} \cdot[S T E]=a \cdot E_{g} \cdot \sigma_{n} \cdot I_{t h}^{n} \cdot \tau \cdot x \cdot N_{O} .
$$

As mentioned above, only a fraction of the incident pulse energy (T1) is absorbed by the matter i.e. a big part is reflected by the plasma [11]. As $\mathrm{I}_{\text {th }}$ is the absorbed intensity threshold beyond which a glass modification occurs, it is the results of $\mathrm{I}_{\mathrm{th}} / \alpha$ incoming intensity, with $\alpha<1$. Due to focusing and taking into account the absorption length, we have:

$T 1=2.3 \frac{I_{t h}}{\alpha} \cdot \tau \cdot S\left(N A, \frac{I_{t h}}{\alpha}\right) \quad$ where $\mathrm{S}\left(\mathrm{NA}, \mathrm{I}_{\mathrm{th}}\right)$ is the average cross section of the focal volume alike the expression $S\left(N A, \frac{I_{t h}}{\alpha}\right)=\frac{\lambda^{2}}{\pi(N A)^{2}+\frac{I_{t h}}{\alpha} 2 \pi n_{0} n_{2}}$ valid for $\mathrm{NA}>0.1 . \alpha$,

is a priori unknown but assumed constant in this paper. It has been determined by considering that the Eq. 3 is fulfilled with the minimum energy $\mathrm{T} 1=0.1 \mu \mathrm{J}$ in pure silica. We found $\alpha=23 \%$. This appears consistent with timeresolved absorption measurements performed in similar experimental conditions [33].

Eq. 3 itself has been solved by taking into account the data available in the literature for the glasses considered in this paper and by solving the time dependent Fourier equation after a time long enough after the pulse. More details will be published elsewhere. Eq. 3 is thus the intersection of the two curves types of curves plotted in Fig. 5. This graph compares the temperature dependence of the relaxation time for the different sample compositions of this paper based on viscosity measurements and shear modulus data [54] change and the cooling profile modeling after single pulse irradiation [53]. For pure silica, the estimate of the fictive temperature is thus around $2490^{\circ} \mathrm{C}$. If we decrease the incident pulse energy, there is of course no more intersection, but if we increase the pulse energy, the fictive temperature decreases slightly to $2250^{\circ} \mathrm{C}$ from 0.1 to $0.3 \mu \mathrm{J}$.

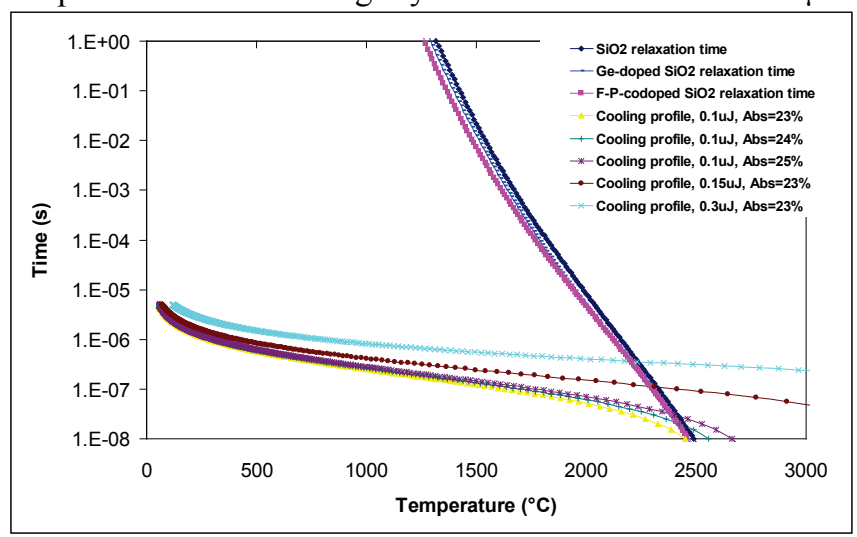

Fig. 5: This graph compares the glass relaxation time and the cooling profile after single pulse femtosecond laser irradiation for pure and doped silica. The cooling profiles with varying incident energy are for pure silica only.

Now, if we consider the case of Ge doping at a level of 1,5 at $\%$ in silica, specific heat capacity increases by less than $2 \%$ [55], viscosity decreases by less than $3 \%$ based on our own measurements and Ref. [56], shear modulus decreases by less than $4 \%$ [54]. The thermal diffusivity is also increasing by not more than $2 \%$. The same is for $\mathrm{P}$ and $\mathrm{F}$ 
doping [57]. Therefore, the critical temperature for structural modification as defined above is expected not to change significantly (less than $50^{\circ} \mathrm{C}$ ) with doping and thus the corresponding energy density appearing in Eq. 4, from those ones we can deduce the change in intensity threshold. We have $\frac{I_{t h, G e}}{I_{t h, S i}}=\left(\frac{E g_{S i} \sigma_{S i} I_{T 1, S i}}{E g_{G e} \sigma_{G e}}\right)^{1 / 5}$

The band gap seems to significantly decrease as the STE localize on Ge atoms even with the weak doping used in this paper [58]. As a result, the band gap decreases from $9 \mathrm{eV}$ to $7.5 \mathrm{eV}$ i.e. by about $17 \%$. On the other hand, the frequency-based spectral interferometry measurements indicate that the STE concentration increases by about a factor 3 [58]. Here we used the multiphoton cross-sections for pure silica (6 photons absorption) and Ge-doped silica (5 photons absorption) that we measured using plasma density measurements in similar experimental conditions $\left(\sigma_{\mathrm{Ge}}\right.$ $=1.810^{-55} \mathrm{~s}^{-1} \mathrm{~cm}^{10} \mathrm{~W}^{-5}$ and $\left.\sigma_{\mathrm{Si}}=4.510^{-69} \mathrm{~s}^{-1} \mathrm{~cm}^{12} \mathrm{~W}^{-6}[25]\right)$ and the silica intensity threshold corresponding to $\mathrm{T} 1$ threshold in silica $\left(\approx 15 \mathrm{TW} / \mathrm{cm}^{2}\right)$. As a result, the variation of the intensity threshold is less than $10 \%$. Further, as the cross section of the focal volume is not varying significantly on doping, we deduce that T1 is not varying either. This is thus consistent with the observations. The ratios calculated for other doped silica leads to the same conclusions: the energy thresholds for glass structural change are about the same. As a matter of fact, comparing relaxation time and cooling curve in doped samples, see Fig. 5 assuming the same proportion of pulse energy absorption, leads to a pulse energy threshold of $0.10 \mu \mathrm{J}$ for Ge doped sample, of $0.095 \mu \mathrm{J}$ of $\mathrm{P}$ and $\mathrm{F}$-doped samples. The corresponding fictive temperatures are $2490^{\circ} \mathrm{C}$ and $2450^{\circ} \mathrm{C}$ respectively.

\section{T2 interpretation}

In Ref. [59] the threshold of voids formation (called T3) in glasses was found to be reciprocal to the concentration of glass former. In addition correlations between glass density, glass transition temperature and young modulus with the voids formation threshold have been revealed. However we do not observe such trends for none of those properties. Indeed $\mathrm{T} 2$ threshold exhibits a totally different origin than T1 or T3 (voids formation). It is the onset of a strong linear birefringence based on the appearance of a long range microstructure i.e. the assembly of nanoplans that sometimes organize in nanogratings [8]. Nanoplanes (a few nm thick) are composed by porous matter [6] that on the average has a lower atomic density than the surrounding glass. They are formed as a result of glass decomposition with releasing oxygen into pores [60]. The appearance of nanoplanes, the orientation of which being determined by laser polarization seems related whatever the interpretation (nanoplasma [61] or phonon-plasmon interference [62]), to plasma density or energy increase significantly larger than the average electron density. It has been noted that it needs several pulses for their observation, the number depending on the pulse energy. On the other hand, we have observed nanogratings and related porous nanoplanes at a repetition rate as low as $1 \mathrm{kHz}$ so with pulses without other relation than some information "written" in the glass. It is therefore clear that the plasma micro-structure is recorded in the glass and that the corresponding information seeds the plasma structure in the subsequent pulse. Referring to the relaxation scheme already sketched for T1 interpretation above, we will hypothesize that point defects, even if they are not the most efficient relaxation pathway are good candidates for recording medium. As a matter of fact, decomposition of silica leads to SiODC(II) defect formation [63], which may behave as trapping centers and then to electron source for the next pulse. Then, in the process of multiphoton ionization, these centers with occupied level in the forbidden gap are expected to be readily ionized in first, contributing to plasma nanostructure formation. As the irradiation is going on, the process self organized into a stable nanostructure.

It is worth noticing that point defects in Ge-doped silica is easier achieved through reduction process $[64,65]$ and Ge itself can easily behave as a trapping centers forming $\mathrm{Ge}(1)$ centers [66]. We have to note also that GeODC(II), inherent to Ge doped silica is an electron source that gives rise to Ge(1) through UV absorption. The same properties stands for phosphorus doped silica [66] but not for fluorine doping, which is also recognized for hardening the glass against the ionizing radiation [67]. On the other hand, decomposition of the glass that depends on the bound energies is easier with $\mathrm{Ge}$ and $\mathrm{P}$ but not with $\mathrm{F}$ doping.

Finally, as we have seen for $\mathrm{T} 1$, the electron density or energy in the plasma seems not being the step that determines the doping dependence of the nanoplanes formation, it is rather the ability of the glass to be decomposed and to record the plasma density spatial structure. In such a way, the branching ratio for STE's to transform into point defects is larger for $\mathrm{Ge}$ and $\mathrm{P}$ than for $\mathrm{F}$. The number of pulses of a given energy is thus varying accordingly and thus the energy for a given number of pulses.

On the other hand, as the energy required for decomposing Ge-doped silica is less than for pure silica, the onset energy for nanoplanes appearance is smaller. Similar remarks stand for P-doping. On the contrary, doping silica with fluorine reduces defects concentrations and the structural disorder [39, 68], this result in a more difficult glass decomposition and thus a higher T2 threshold.

More quantitatively, we may consider that birefringence is always detected above the same quantity (quoted as [B]). This minimal quantity is certainly proportional to an amount of glass decomposition which is itself proportional to the branching ratio $(\varepsilon)$ from STE and to STE concentration ([STE]) produced by a series of pulses $(\mathrm{N})$. We may write $[\mathrm{B}] \sim \mathrm{N} \cdot \varepsilon \cdot[\mathrm{STE}]$. On the other hand, $[\mathrm{STE}] \sim \sigma \cdot \mathrm{I}^{\mathrm{k}}$ where $\mathrm{k}$ is the number of photon in the MPI process. So, finally we get:

$$
\frac{I_{t h 2, G e}}{I_{t h 2, S i}}=\left(\frac{N_{S i} \varepsilon_{S i} \sigma_{S i} I_{t h 2, S i}}{N_{G e} \varepsilon_{G e} \sigma_{G e}}\right)^{1 / 5} \text { with } \mathrm{I}_{\mathrm{th} 2, \mathrm{Si}} \approx 40 \mathrm{TW} / \mathrm{cm}^{2} .
$$

Again referring to the focal volume cross section that is nearly invariant on doping and to the Table 2, we find that the left term of the above equation reaches about 0.5 . We deduce that $\mathrm{N} \cdot \varepsilon$ ratio reaches about 0.1 , meaning that the branching ratio is in the order of 10 in favor of Ge if we assume that the number of pulses is the same for both glasses.

\section{Conclusion}


We have investigated electronic plasma induced by a focused single femtosecond laser pulse in various doped silica glasses. The measured mean value $N_{S T E}$ is of the order of $N_{S T E} \sim 2.10^{19} \mathrm{~cm}^{-3}$ in $\mathrm{P}$ or Ge-doped silica and at least two times smaller in pure silica or in F-doped silica. The mean trapping time of electrons excited in the conduction band was found to be significantly lower in Ge-doped silica $\left(\tau_{t r G e} \approx 75 \pm 5 \mathrm{fs}\right)$ and $\mathrm{P} / \mathrm{F}$ co-doped silica $\left(\tau_{t r P} \approx 85 \pm 5 \mathrm{fs}\right)$ when compared to pure silica or F-doped silica $\left(\tau_{t r S i} \approx 155\right.$ $\pm 5 \mathrm{fs}$ ). At our concentration level, this indicates that the plasma properties are determined by the presence of easily ionisable states such as the presence of $\mathrm{Ge}$ or $\mathrm{P}$ atoms in the glass network. Therefore, we suggest that in Ge-doped and P-doped silica there exist an additional trapping pathway that leads to a significantly faster trapping and a higher plasma density when compared to un-doped silica.

We show that strong birefringence, which is not observed to this date in glasses other than pure silica, appears also in doped silica. The first threshold (i.e. permanent isotropic index change) is not significantly dependent on the chemical composition, i.e. doping, in contrast to the second threshold (i.e. permanent linear birefringence). This is explained by noting that isotropic index change at low pulse energies arising from glass modification allowed when the glass is heated a time long enough for restructuration (larger than the relaxation time) and that this requested time does not change much at our doping level. On the contrary, the second threshold is related to nanoplanes of decomposed silica and produced through a mechanism that requires point or extended defects to be produced by the plasma and record its structure. The efficiency of this mechanism is thus determined by the ability of the glass to produce point defects and the energy for glass decomposition.

From a practical point of view, it appears that silica doped with small amounts of either germanium or phosphorous is a better choice for birefringence tailoring than pure silica glass, since the processing window is wider. In contrast, it is the reverse influence for fluorine doping. It is thus judicious to use Ge or P doped silica for discrete optical device fabrication when birefringence is required, such as for a retardation plate or Fresnel lens. As the retardance can be accumulated over many layers, quarter and half waveplates can be easily realized over a typical $100 \mathrm{mi}-$ crons thickness. F-doped silica is well adapted for devices based on isotropic index change such as 3D waveguides or volume Bragg gratings. Indeed, the pulse energy processing window is quite large (from 0.1 up to 1.20 $\mu \mathrm{J} /$ pulse) which allows us to tailor the photo-induced index change profile. In addition the phase change can be as high as $-\pi / 2$. This means that we can realize phase lens such as annular phase ring in two or three layers (i.e. less than 150 microns thickness).

\section{Acknowledgments and Appendixes}

This work has been performed in the frame of FLAG (Femtosecond Laser Application in Glasses) consortium project with the support of several organisations: the Agence Nationale pour la Recherche (ANR-09-BLAN0172-01), the RTRA Triangle de la Physique (Réseau Thématique de Recherche Avancée, 2008-056T), the Es- sonne administrative Department (ASTRE2007), the Ministry of the Foreign Affairs (PHC Alliance) and FP7PEOPLE-IRSES e-FLAG 247635.

\section{References}

[1] L. Sudrie, M. Franco, B. Prade, and A. Mysyrowicz, Optics Communications 171 (1999) 279.

[2] B. Poumellec, M. Lancry, J. Poulin, and S. Ani-Joseph, Optics Express 16 (2008) 18354.

[3] B. Poumellec, L. Sudrie, M. Franco, B. Prade, and A. Mysyrowicz, Optics Express 11 (2003) 1070.

[4] P. Kazansky, W. Yang, E. Bricchi, J. Bovatsek, A. Arai, Y. Shimotsuma, K. Miura, and K. Hirao, Applied Physics Letters 90 (2007) 151120.

[5] J. Qiu, K. Miura, and K. Hirao, Journal of NonCrystalline Solids 354 (2008) 1100.

[6] M. Lancry, F. Brisset, and B. Poumellec, in Bragg Gratings, Photosensitivity, and Poling in Glass Waveguides, OSA Technical Digest (CD), Optical Society of America, 2010.

[7] M. Kaempfe, G. Seifert, K. Berg, H. Hofmeister, and H. Graener, The European Physical Journal D-Atomic, Molecular, Optical and Plasma Physics 16 (2001) 237.

[8] Y. Shimotsuma, P. Kazansky, J. Qiu, and K. Hirao, Physical review letters 91 (2003) 247405.

[9] M. Ams, G. Marshall, P. Dekker, M. Dubov, V. Mezentsev, I. Bennion, and M. Withford, IEEE Journal of Selected Topics in Quantum Electronics 14 (2008) 1370.

[10]K. Itoh, W. Watanabe, S. Nolte, and C. Schaffer, MRS bulletin 31 (2006) 620.

[11]F. Quéré, S. Guizard, and P. Martin, EPL (Europhysics Letters) 56 (2001) 138.

[12] V. Temnov, K. Sokolowski-Tinten, P. Zhou, A. ElKhamhawy, and D. von der Linde, Physical review letters 97 (2006) 237403.

[13] S. Guizard, A. Belsky, J. Gaudin, G. Geoffroy, P. Martin, G. Petite, A. Philippov, and B. Yatsenko, physica status solidi (c) 2 (2005) 223.

[14]J. Chan, T. Huser, S. Risbud, and D. Krol, Optics Letters 26 (2001) 1726.

[15] S. Guizard, P. Martin, G. Petite, P. D'Oliveira, and P. Meynadier, Journal of Physics: Condensed Matter 8 (1996) 1281

[16]H. Sun, S. Juodkazis, M. Watanabe, S. Matsuo, H. Misawa, and J. Nishii, J. Phys. Chem. B 104 (2000) 3450.

[17]K. Davis, K. Miura, N. Sugimoto, and K. Hirao, Optics letters 21 (1996) 1729.

[18]K. Hirao and K. Miura, Journal of Non-Crystalline Solids 239 (1998) 91.

[19] S. Nolte, M. Will, J. Burghoff, and A. Tuennermann, Applied Physics A: Materials Science \& Processing 77 (2003) 109.

[20] S. Mao, F. Quéré, S. Guizard, X. Mao, R. Russo, G. Petite, and P. Martin, Applied Physics A: Materials Science \& Processing 79 (2004) 1695.

[21]P. Martin, S. Guizard, P. Daguzan, G. Petite, P. D'Oliveira, P. Meynadier, and M. Perdrix, Physical Review B 55 (1997) 5799.

[22]T. Kato, Y. Suetsugu, and M. Nishimura, Optics Letters 20 (1995) 2279. 
[23]P. Bachmann, W. Hermann, H. Wehr, and D. Wiechert, Applied optics 25 (1986) 1093.

[24]G. Scherer, Journal of Non-Crystalline Solids 38 (1980) 201.

[25]M. Lancry, N. Groothoff, B. Poumellec, S. Guizard, N. Fedorov, and J. Canning, Physics Review B In Press (2011) [26]D. Griscom, Physical Review B 40 (1989) 4224.

[27]C. Itoh, K. Tanimura, and N. Itoh, Journal of Physics C: Solid State Physics 21 (1988) 4693.

[28]C. Itoh, K. Tanimura, N. Itoh, and M. Itoh, Physical Review B 39 (1989) 11183.

[29]N. Itoh, T. Shimizu-Iwayama, and T. Fujita, Journal of Non-Crystalline Solids 179 (1994) 194.

[30]A. Trukhin, NATO science series II, in: D. Griscom, G. Pacchioni, L. Skuja (Eds.), Mathematics, Physics and Chemistry, Defects in SiO 2 (2000)

[31]M. Lancry, N. Groothoff, S. Guizard, W. Yang, B. Poumellec, P. Kazansky, and J. Canning, Journal of NonCrystalline Solids 355 (2009) 1057.

[32]K. Ishii, A. Takami, and Y. Ohki, Journal of Applied Physics 81 (1997) 1470.

[33]A. Couairon, L. Sudrie, M. Franco, B. Prade, and A. Mysyrowicz, Physical Review B 71 (2005) 125435.

[34]E. Friebele and D. Griscom, Vol. 319, 1986.

[35]M. Fujimaki, T. Watanabe, T. Katoh, T. Kasahara, N. Miyazaki, Y. Ohki, and H. Nishikawa, Physical Review B 57 (1998) 3920.

[36]D. Griscom, E. Friebele, K. Long, and J. Fleming, Journal of Applied Physics 54 (1983) 3743.

[37]J. Weil, Physics and Chemistry of Minerals 10 (1984) 149.

[38]G. Pacchioni, D. Erbetta, D. Ricci, and M. Fanciulli, J. Phys. Chem. B 105 (2001) 6097.

[39]L. Skuja, K. Kajihara, Y. Ikuta, M. Hirano, and H. Hosono, Journal of Non-Crystalline Solids 345 (2004) 328.

[40]H. Hosono, Y. Ikuta, T. Kinoshita, K. Kajihara, and M. Hirano, Physical review letters 87 (2001) 175501.

[41]K. Kajihara, Y. Ikuta, M. Oto, M. Hirano, L. Skuja, and H. Hosono, Nuclear Instruments and Methods in Physics Research Section B: Beam Interactions with Materials and Atoms 218 (2004) 323.

[42]K. Arai, H. Imai, J. Isoya, H. Hosono, Y. Abe, and H. Imagawa, Physical Review B 45 (1992) 10818.

[43] S. Girard, C. Marcandella, G. Origlio, Y. Ouerdane, A. Boukenter, and J. Meunier, Journal of Non-Crystalline Solids 355 (2009) 1089.

[44]H. Hosono, M. Mizuguchi, H. Kawazoe, and T. Ogawa, Applied Physics Letters 74 (1999) 2755.

[45]C. Schaffer, A. Brodeur, and E. Mazur, Measurement Science and Technology 12 (2001) 1784.

[46]M. Lancry, B. Poumellec, and M. Douay, Journal of Non-Crystalline Solids 355 (2009) 1062.

[47]J. Chan, T. Huser, S. Risbud, and D. Krol, Applied Physics A: Materials Science \& Processing 76 (2003) 367. [48]H. Hosono, K. Kawamura, S. Matsuishi, and M. Hirano, Nuclear Inst. and Methods in Physics Research, B 191 (2002) 89.

[49]S. Guizard, P. Martin, G. Petite, P. D'Oliveira, and P. Meynadier, Journal of Physics: Condensed Matter 8 (1996) 1281.

[50]L. Skuja, M. Hirano, H. Hosono, and K. Kajihara, Physica Status Solidi (c) 2 (2005) 15.
[51]M. Lancry, E. Régnier, and B. Poumellec, Progress in Materials Science (2011)

[52]A. Tool, Journal of the American Ceramic Society 29 (1946) 240.

[53] S. Eaton, H. Zhang, P. Herman, F. Yoshino, L. Shah, J. Bovatsek, and A. Arai, Optics Express 13 (2005) 4708.

[54]M. Yamane and J. Mackenzie, Journal of NonCrystalline Solids 15 (1974) 153.

[55]P. Richet, D. de Ligny, and E. Westrum, Journal of Non-Crystalline Solids 315 (2003) 20.

[56]K. Tajima, M. Tateda, and M. Ohashi, Journal of Lightwave Technology 12 (1994) 411.

[57]M. Kyoto, Y. Ohoga, S. Ishikawa, and Y. Ishiguro, Journal of Materials Science 28 (1993) 2738.

[58]M. Lancry and B. Poumellec, Femtosecond Laser Microfabrication (2009)

[59]T. Hashimoto, S. Juodkazis, and H. Misawa, New Journal of Physics 9 (2007) 253.

[60]M. Lancry, K. Cook, J. Canning, and B. Poumellec, in The International Quantum Electronics Conference (IQEC)/The Conference on Lasers and Electro-Optics (CLEO) Pacific Rim, Sydney, Australia, 2011.

[61]P. Rajeev, M. Gertsvolf, C. Hnatovsky, E. Simova, R. Taylor, P. Corkum, D. Rayner, and V. Bhardwaj, Journal of Physics B: Atomic, Molecular and Optical Physics 40 (2007) S273.

[62]P. Kazansky, E. Bricchi, Y. Shimotsuma, and K. Hirao, IEEE, 2008, p. 1.

[63]M. Lancry, B. Poumellec, W. Yang, and B. Bourguignon, Optics Express In Press (2011)

[64]R. Araujo, Journal of Non-Crystalline Solids 197 (1996) 164.

[65]M. Schurman and M. Tomozawa, Journal of NonCrystalline Solids 202 (1996) 93.

[66]T. Tsai, E. Friebele, and D. Griscom, Optics letters 18 (1993) 935.

[67]K. Sanada, N. Shamoto, and K. Inada, Journal of NonCrystalline Solids 179 (1994) 339.

[68]K. Saito and A. Ikushima, Journal of Applied Physics 91 (2002) 4886.

(Received: July 27, 2011, Accepted: May 05, 2012) 